\title{
Study of the Formation of the First Aromatic Rings in the Pyrolysis of Cyclopentene
}

\author{
Olivier Herbinet ${ }^{a, b,}{ }^{,}$, Anne Rodriguez ${ }^{a, b}$, Benoit Husson ${ }^{a, b}$, Frederique Battin- \\ Leclerc $^{\mathrm{a}, \mathrm{b}}$, Zhandong Wang ${ }^{\mathrm{c}}$, Zhanjun Cheng ${ }^{c}$, Fei $\mathrm{Qi}^{\mathrm{c}}$ \\ aUniversité de Lorraine, Laboratoire Réactions et Génie des Procédés, UMR 7274, Nancy, F-54000, \\ France \\ ${ }^{\mathrm{b}} \mathrm{CNRS}$, Laboratoire Réactions et Génie des Procédés, UMR 7274, Nancy, F-54000, France \\ ${ }^{c}$ National Synchrotron Radiation Laboratory, University of Science and Technology of China, Hefei, \\ Anhui 230029, P. R. China.
}

\section{Supplemental data}


Figure S1: Schematic diagram of the instruments including the jet-stirred reactor and in (a) the synchrotron photoionization mass spectrometer and in (b) the laser photoionization mass spectrometer.

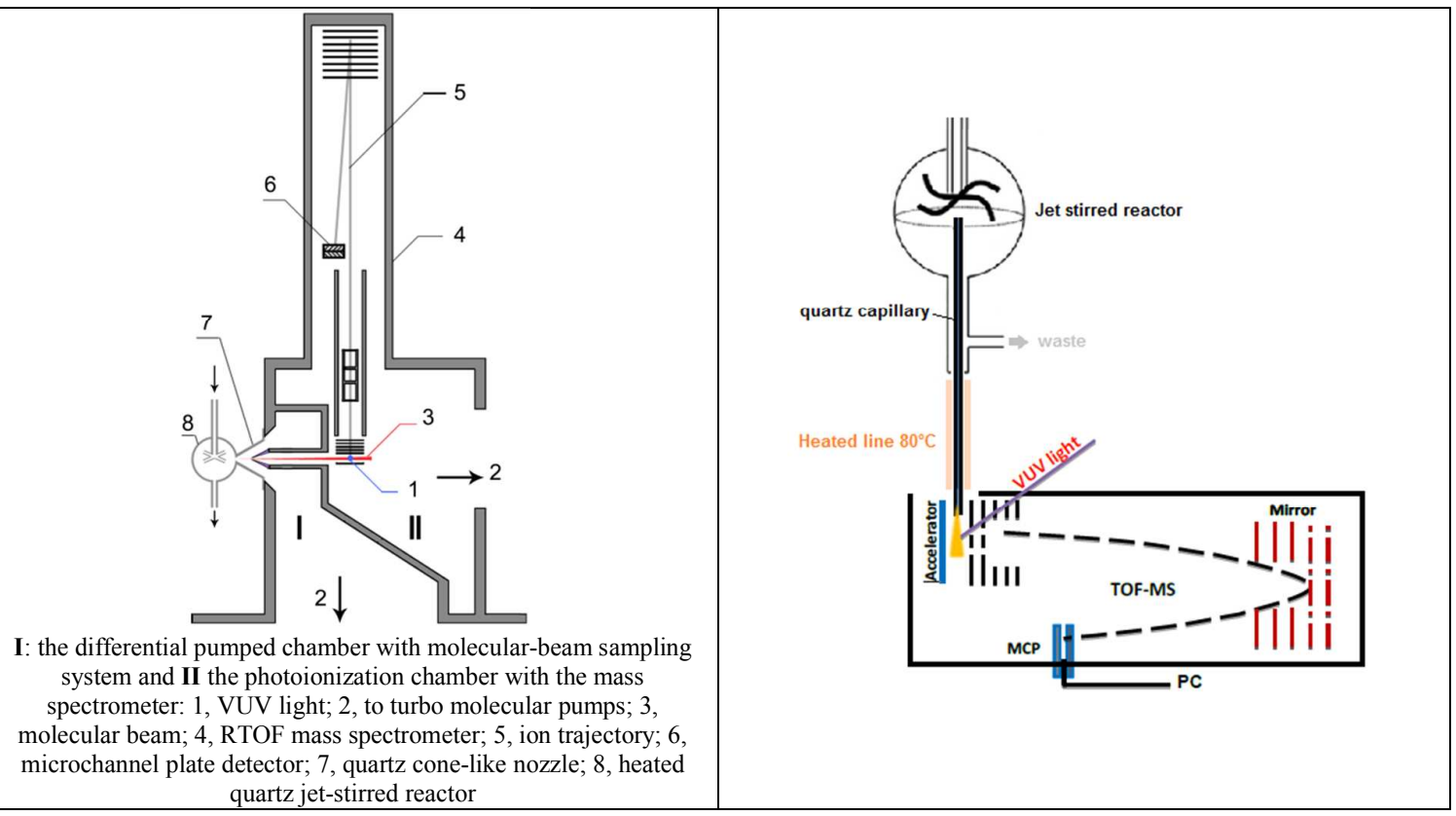


Table S1: Adiabatic ionization energies calculated using software Gaussian [1]. Calculations were performed at the CBS-QB3 level of theory [2]. Comparison with literature data [3].

\begin{tabular}{|c|c|c|c|}
\hline Species & Structure & Calculated AIEs (eV) & Literature data (eV) \\
\hline cyclopentadienyl radical & & $8.73^{a}, 8.48^{b}$ & $8.41,8.56,8.7$ \\
\hline$m / z 78$ & & & \\
\hline benzene & & 9.26 & 9.24 \\
\hline fulvene & & 8.43 & 8.36 \\
\hline 1,3-hexadiyne & & 9.31 & $9.25,9.41$ \\
\hline 1,4-hexadiyne & & 9.66 & $9.50-9.75$ \\
\hline 1,5-hexadiyne & & 9.97 & $9.9-10.0$ \\
\hline 2,4-hexadiyne & & 8.82 & $8.9-9.0$ \\
\hline trans-1,3-hexadien-5-yne & & 8.61 & $9.2-9.5$ \\
\hline cis-1,3-hexadien-5-yne & & 8.65 & $9.2-9.5$ \\
\hline 1,5-hexadien-3-yne & & 8.61 & 8.5 \\
\hline
\end{tabular}

${ }^{a}$ singlet state for the cation. ${ }^{b}$ triplet state for the cation. 
Table S2: Kinetic parameters used for the formation of toluene from the recombination of cyclopentadienyl and ethyl radicals as shown in Figure $\mathrm{S} 2$ (units: $\mathrm{cm}^{3}, \mathrm{~mol}, \mathrm{~s}, \mathrm{cal}$ ).

\begin{tabular}{|c|c|c|c|c|}
\hline Reaction & $\mathrm{A}$ & $\mathrm{n}$ & Ea & ref \\
\hline \multicolumn{5}{|l|}{ Combination } \\
\hline $\mathrm{C}_{5} \mathrm{H}_{5}+\mathrm{C}_{2} \mathrm{H}_{5} \leftrightarrows \mathrm{ECPD}$ & $8.34 \times 10^{15}$ & -0.70 & -0.50 & 1 \\
\hline \multicolumn{5}{|l|}{ Unimolecular Initiations } \\
\hline $\mathrm{ECPD} \leftrightarrows \mathrm{R} 1 \mathrm{EACPD}+\mathrm{H}$ & $2.5 \times 10^{15}$ & 0 & $78.7 \times 10^{3}$ & 2 \\
\hline $\mathrm{R} 2 \mathrm{ECPD}+\mathrm{H} \leftrightarrows \mathrm{ECPD}$ & $1.00 \times 10^{14}$ & 0 & 0 & 3 \\
\hline $\mathrm{R} 3 \mathrm{ECPD}+\mathrm{H} \leftrightarrows \mathrm{ECPD}$ & $1.00 \times 10^{14}$ & 0 & 0 & 3 \\
\hline \multicolumn{5}{|l|}{ H-atom abstractions } \\
\hline $\mathrm{ECPD}+\mathrm{H} \leftrightarrows \mathrm{R} 1 \mathrm{ECPD}+\mathrm{H}_{2}$ & $1.4 \times 10^{13}$ & 0 & $2.0 \times 10^{3}$ & 4 \\
\hline $\mathrm{ECPD}+\mathrm{H} \leftrightarrows \mathrm{R} 2 \mathrm{ECPD}+\mathrm{H}_{2}$ & $4.5 \times 10^{6}$ & 2 & $5.0 \times 10^{3}$ & 5 \\
\hline $\mathrm{ECPD}+\mathrm{H} \leftrightarrows \mathrm{R} 3 \mathrm{ECPD}+\mathrm{H}_{2}$ & $2.9 \times 10^{7}$ & 2 & $7.7 \times 10^{3}$ & 5 \\
\hline $\mathrm{ECPD}+\mathrm{CH}_{3} \leftrightarrows \mathrm{R} 1 \mathrm{ECPD}+\mathrm{CH}_{4}$ & 590 & 2.9 & $5.06 \times 10^{3}$ & 6 \\
\hline $\mathrm{ECPD}+\mathrm{CH}_{3} \leftrightarrows \mathrm{R} 2 \mathrm{ECPD}+\mathrm{CH}_{4}$ & $1.0 \times 10^{11}$ & 0 & $9.6 \times 10^{3}$ & 5 \\
\hline $\mathrm{ECPD}+\mathrm{CH}_{3} \leftrightarrows \mathrm{R} 3 \mathrm{ECPD}+\mathrm{CH}_{4}$ & $0.75 \times 10^{-7}$ & 6 & $5.8 \times 10^{3}$ & 7 \\
\hline $\mathrm{ECPD}+\mathrm{C}_{3} \mathrm{H}_{5} \leftrightarrows \mathrm{R} 1 \mathrm{ECPD}+\mathrm{C}_{3} \mathrm{H}_{6}$ & 96 & 3.14 & $11.5 \times 10^{3}$ & 8 \\
\hline $\mathrm{ECPD}+\mathrm{C}_{3} \mathrm{H}_{5} \leftrightarrows \mathrm{R} 2 \mathrm{ECPD}+\mathrm{C}_{3} \mathrm{H}_{6}$ & $4.0 \times 10^{1}$ & 3.3 & $18.17 \times 10^{3}$ & 5 \\
\hline $\mathrm{ECPD}+\mathrm{C}_{3} \mathrm{H}_{5} \leftrightarrows \mathrm{R} 3 \mathrm{ECPD}+\mathrm{C}_{3} \mathrm{H}_{6}$ & $4.0 \times 10^{1}$ & 3.3 & $19.84 \times 10^{3}$ & 5 \\
\hline $\mathrm{ECPD}+\mathrm{C}_{5} \mathrm{H}_{5} \leftrightarrows \mathrm{R} 1 \mathrm{ECPD}+\mathrm{C}_{5} \mathrm{H}_{6}$ & 96 & 3.14 & $11.5 \times 10^{3}$ & 8 \\
\hline $\mathrm{ECPD}+\mathrm{C}_{5} \mathrm{H}_{5} \leftrightarrows \mathrm{R} 2 \mathrm{ECPD}+\mathrm{C}_{5} \mathrm{H}_{6}$ & $8.0 \times 10^{1}$ & 3.3 & $18.17 \times 10^{3}$ & 5 \\
\hline \multicolumn{4}{|l|}{ Isomerization } & 5 \\
\hline $\mathrm{R} 1 \mathrm{ECPD} \leftrightarrows \mathrm{R} 2 \mathrm{ECPD}$ & $3.0 \times 10^{13}$ & 0 & $50.4 \times 10^{3}$ & 9 \\
\hline $\mathrm{R} 3 \mathrm{ECPD} \leftrightarrows \mathrm{R} 1 \mathrm{ECPD}$ & $1.92 \times 10^{9}$ & 1 & $32.5 \times 10^{3}$ & 10 \\
\hline \multicolumn{5}{|l|}{ Reactions of decomposition } \\
\hline $\mathrm{R} 3 \mathrm{ECPD} \leftrightarrows \mathrm{C}_{2} \mathrm{H}_{4}+\mathrm{C}_{5} \mathrm{H}_{5}$ & $3.3 \times 10^{13}$ & 0 & $22.5 \times 10^{3}$ & 11 \\
\hline $\mathrm{R} 2 \mathrm{ECPD} \leftrightarrows \mathrm{R} 4 \mathrm{ECPD}$ & $1.4 \times 10^{13}$ & 0 & $17.4 \times 10^{3}$ & 12 \\
\hline $\mathrm{R} 2 \mathrm{ECPD} \leftrightarrows \mathrm{R} 4 \mathrm{ECPD}$ & $4.0 \times 10^{13}$ & 0 & $35.5 \times 10^{3}$ & 11 \\
\hline toluene $+\mathrm{H} \leftrightarrows \mathrm{R} 4 \mathrm{ECPD}$ & $3.2 \times 10^{13}$ & 0 & $3.2 \times 10^{3}$ & 13 \\
\hline
\end{tabular}

\footnotetext{
${ }^{1}$ as cyclopentadienyl $+\mathrm{CH}_{3}[4]$.

${ }^{2}$ as 1,3-cyclopentadiene $=$ cyclopentadienyl $+\mathrm{H}[5], \mathrm{A} / 2$.

${ }^{3}$ Exgas correlation for recombination with $\mathrm{H}$-atoms [6].

${ }^{4}$ as for 1,3 -cyclopentadiene $+\mathrm{H}=$ cyclopentadienyl $+\mathrm{H}_{2}[7], \mathrm{A} / 2$.

${ }^{5}$ Exgas correlation for $\mathrm{H}$-atom abstractions [6].

${ }^{6}$ as for 1,3-cyclopentadiene $+\mathrm{CH}_{3}=$ cyclopentadienyl $+\mathrm{CH}_{4}[8]$.

${ }^{7}$ as $\mathrm{C}_{2} \mathrm{H}_{6}+\mathrm{CH}_{3}=\mathrm{C}_{2} \mathrm{H}_{5}+\mathrm{CH}_{4}[9], \mathrm{A} / 2$.

${ }^{8}$ as for 1,3 -cyclopentadiene $+\mathrm{H}=$ cyclopentadienyl $+\mathrm{H}_{2}[7], \mathrm{A} / 2$.

${ }^{9}$ as for methylcyclopentadienyl radical $=5$-methylene-1,3-cyclopentadiene radical [10].

${ }^{10}$ Exgas correlation for isomerizations [6].

${ }^{11}$ Exgas correlation for $\beta$-scission [6]

${ }^{12}$ as for 5-methylene-1,3-cyclopentadiene $=2,4$-cyclohexadien-1-yl radical [10].

${ }^{13}$ as benzene $+\mathrm{H}=2,4$-cyclohexadien-1-yl radical [11].
} 
Table S3: Kinetic parameters used for the formation of toluene from the addition of the cyclopentadienyl radical to propene as shown in Figure $S 3$ (units: $\mathrm{cm}^{3}, \mathrm{~mol}, \mathrm{~s}, \mathrm{cal}$ ).

\begin{tabular}{|c|c|c|c|c|}
\hline Reaction & A & $\mathrm{n}$ & $\mathrm{Ea}$ & ref \\
\hline \multicolumn{5}{|l|}{ Addition } \\
\hline $\mathrm{C}_{5} \mathrm{H}_{5}+$ propene $\leftrightarrows \mathrm{C} 5 \mathrm{H} 5 @ \mathrm{C} 3 \mathrm{H} 6 \mathrm{I}$ & $1.56 \times 10^{3}$ & 2.53 & $20.0 \times 10^{3}$ & 1 \\
\hline \multicolumn{5}{|l|}{ Cyclo-addition } \\
\hline C5H5@C3H6I $\rightarrow$ C8H11-C4 & $3.10 \times 10^{12}$ & 0 & $10.4 \times 10^{3}$ & [12] \\
\hline reverse & $1.60 \times 10^{13}$ & 0 & $27.1 \times 10^{3}$ & [12] \\
\hline \multicolumn{5}{|l|}{ Decomposition } \\
\hline $\mathrm{C} 8 \mathrm{H} 11-\mathrm{C} 4 \rightarrow \mathrm{C} 8 \mathrm{H} 11-\mathrm{C} 7$ & $1.4 \times 10^{13}$ & 0 & $24.6 \times 10^{3}$ & {$[12]$} \\
\hline reverse & $2.7 \times 10^{13}$ & 0 & $59.0 \times 10^{3}$ & {$[12]$} \\
\hline $\begin{array}{l}\mathrm{C} 8 \mathrm{H} 11-\mathrm{C} 7 \leftrightarrows \mathrm{C} 7 \mathrm{H} 8-\mathrm{c} 7+\mathrm{CH}_{3} \\
\text { Isomerization }\end{array}$ & $1.0 \times 10^{13}$ & 0 & $31.0 \times 10^{3}$ & \\
\hline $\mathrm{R} 1 \mathrm{ECPD} \leftrightarrows \mathrm{R} 2 \mathrm{ECPD}$ & $3.0 \times 10^{13}$ & 0 & $50.4 \times 10^{3}$ & [13] \\
\hline
\end{tabular}

${ }^{1}$ estimation from the addition of allyl to propene [8].

${ }^{2}$ Exgas correlation for $\beta$-scission [6] 
Table S4: Kinetic parameters used for the formation of styrene from the cyclopentadienyl and allyl radicals as shown in Figure $S 4$ (units: $\mathrm{cm}^{3}, \mathrm{~mol}, \mathrm{~s}$, cal).

\begin{tabular}{|c|c|c|c|c|}
\hline Reaction & $\mathrm{A}$ & $\mathrm{n}$ & Ea & ref \\
\hline \multicolumn{5}{|l|}{ Combination } \\
\hline $\mathrm{C}_{5} \mathrm{H}_{5}+\mathrm{C}_{3} \mathrm{H}_{5} \leftrightarrows \mathrm{ACPD}$ & $8.34 \times 10^{15}$ & -0.70 & -0.50 & 1 \\
\hline \multicolumn{5}{|l|}{ Unimolecular Initiations } \\
\hline $\mathrm{ACPD} \leftrightarrows \mathrm{R} 1 \mathrm{ACPD}+\mathrm{H}$ & $2.5 \times 10^{15}$ & 0 & $78.7 \times 10^{3}$ & 2 \\
\hline $\mathrm{ACPD} \leftrightarrows \mathrm{R} 2 \mathrm{ACPD}+\mathrm{H}$ & $1.26 \times 10^{15}$ & 0 & $82.47 \times 10^{3}$ & 3 \\
\hline \multicolumn{5}{|l|}{ H-atom abstractions } \\
\hline $\mathrm{ACPD}+\mathrm{H} \leftrightarrows \mathrm{R} 1 \mathrm{ACPD}+\mathrm{H}_{2}$ & $1.4 \times 10^{13}$ & 0 & $2.0 \times 10^{3}$ & 4 \\
\hline $\mathrm{ACPD}+\mathrm{H} \leftrightarrows \mathrm{R} 2 \mathrm{ACPD}+\mathrm{H}_{2}$ & $1.13 \times 10^{5}$ & 2.5 & $2.48 \times 10^{3}$ & 5 \\
\hline $\mathrm{ACPD}+\mathrm{CH}_{3} \leftrightarrows \mathrm{R} 1 \mathrm{ACPD}+\mathrm{CH}_{4}$ & 590 & 2.9 & $5.06 \times 10^{3}$ & 6 \\
\hline $\mathrm{ACPD}+\mathrm{CH}_{3} \leftrightarrows \mathrm{R} 2 \mathrm{ACPD}+\mathrm{CH}_{4}$ & 1.47 & 3.5 & $5.68 \times 10^{3}$ & 7 \\
\hline $\mathrm{ACPD}+\mathrm{C}_{3} \mathrm{H}_{5} \leftrightarrows \mathrm{R} 1 \mathrm{ACPD}+\mathrm{C}_{3} \mathrm{H}_{6}$ & 96 & 3.14 & $11.5 \times 10^{3}$ & 8 \\
\hline $\mathrm{ACPD}+\mathrm{C}_{3} \mathrm{H}_{5} \leftrightarrows \mathrm{R} 2 \mathrm{ACPD}+\mathrm{C}_{3} \mathrm{H}_{6}$ & $4.0 \times 10^{1}$ & 3.3 & $18.17 \times 10^{3}$ & 9 \\
\hline $\mathrm{ACPD}+\mathrm{C}_{5} \mathrm{H}_{5} \leftrightarrows \mathrm{R} 1 \mathrm{ACPD}+\mathrm{C}_{5} \mathrm{H}_{6}$ & 96 & 3.14 & $11.5 \times 10^{3}$ & 8 \\
\hline & \multicolumn{4}{|c|}{ Isomerization } \\
\hline $\mathrm{R} 1 \mathrm{ACPD} \leftrightarrows \mathrm{R} 2 \mathrm{ACPD}$ & $3.0 \times 10^{13}$ & 0 & $50.4 \times 10^{3}$ & 10 \\
\hline \multicolumn{5}{|c|}{ Reactions of decomposition to styrene } \\
\hline $\mathrm{R} 2 \mathrm{ACPD} \leftrightarrows \mathrm{R} 3 \mathrm{ACPD}$ & $1.4 \times 10^{13}$ & 0 & $17.4 \times 10^{3}$ & 11 \\
\hline $\mathrm{R} 2 \mathrm{ACPD} \leftrightarrows \mathrm{R} 3 \mathrm{ACPD}$ & $4.0 \times 10^{13}$ & 0 & $35.5 \times 10^{3}$ & 12 \\
\hline Styrene $+\mathrm{H} \leftrightarrows \mathrm{R} 3 \mathrm{ACPD}$ & $3.2 \times 10^{13}$ & 0 & $3.2 \times 10^{3}$ & 13 \\
\hline
\end{tabular}

\footnotetext{
${ }^{1}$ as cyclopentadienyl $+\mathrm{CH}_{3}[4]$.

2 as 1,3-cyclopentadiene $=$ cyclopentadienyl $+\mathrm{H}[5], \mathrm{A} / 2$.

${ }^{3}$ as 1-butene = but-3-en-1-yl + $\mathrm{H}$ [14].

${ }^{4}$ as for 1,3-cyclopentadiene $+\mathrm{H}=$ cyclopentadienyl $+\mathrm{H}_{2}$ [7], $\mathrm{A} / 2$.

${ }^{5}$ as for propene $+\mathrm{H}=$ allyl $+\mathrm{H}_{2}[15], A^{*} 2 / 3$.

${ }^{6}$ as for 1,3-cyclopentadiene $+\mathrm{CH}_{3}=$ cyclopentadienyl $+\mathrm{CH}_{4}$ [8] .

${ }^{7}$ as for propene $+\mathrm{CH}_{3}=$ allyl $+\mathrm{CH}_{4}[15], \mathrm{A}^{*} 2 / 3$.

${ }^{8}$ as for 1,3-cyclopentadiene + allyl = cyclopentadienyl + propene [8].

${ }^{9}$ Exgas correlation for $\mathrm{H}$-atom abstractions by allylic radicals [6]

${ }^{10}$ as for methylcyclopentadienyl radical $=5$-methylene-1,3-cyclopentadiene radical [10].

${ }^{11}$ as for 5-methylene-1,3-cyclopentadiene = 2,4-cyclohexadien-1-yl radical [10].

${ }^{12}$ Exgas correlation for $\beta$-scission [6]

${ }^{13}$ as benzene $+\mathrm{H}=2,4$-cyclohexadien-1-yl radical [11].
} 
Table S5: Kinetic parameters used for the formation of naphthalene from the cyclopentadienyl radical $\left(\mathrm{C}_{5} \mathrm{H}_{5}\right)$ as shown in Figure 8 (units: $\mathrm{cm}^{3}, \mathrm{~mol}, \mathrm{~s}$, cal).

\begin{tabular}{|c|c|c|c|c|}
\hline Reaction & A & $\mathrm{n}$ & Ea & ref \\
\hline \multicolumn{5}{|l|}{ Combination } \\
\hline $\mathrm{C}_{5} \mathrm{H}_{5}+\mathrm{C}_{5} \mathrm{H}_{5} \leftrightarrows \mathrm{DCPD} 1$ & $1.02 \times 10^{13}$ & -0.26 & 0 & {$[8]$} \\
\hline \multicolumn{5}{|l|}{ Unimolecular initiation } \\
\hline $\mathrm{DCPD1} \leftrightarrows \mathrm{R} 1 \mathrm{DCPD}+\mathrm{H}$ & $5.0 \times 10^{15}$ & 0 & $78.7 \times 10^{3}$ & [16] \\
\hline \multicolumn{5}{|l|}{ H-atom abstractions } \\
\hline $\mathrm{DCPD} 1+\mathrm{H} \leftrightarrows \mathrm{R} 1 \mathrm{DCPD}+\mathrm{H}_{2}$ & $2.8 \times 10^{13}$ & 0 & $2.0 \times 10^{3}$ & 1 \\
\hline $\mathrm{DCPD} 1+\mathrm{CH}_{3} \leftrightarrows \mathrm{R} 1 \mathrm{DCPD}+\mathrm{CH}_{4}$ & 1180 & 2.9 & $5.06 \times 10^{3}$ & 2 \\
\hline $\mathrm{DCPD} 1+\mathrm{C}_{3} \mathrm{H}_{5} \leftrightarrows \mathrm{R} 1 \mathrm{DCPD}+\mathrm{C}_{3} \mathrm{H}_{6}$ & 191 & 3.14 & $11.5 \times 10^{3}$ & 3 \\
\hline $\mathrm{DCPD} 1+\mathrm{C}_{5} \mathrm{H}_{5} \leftrightarrows \mathrm{R} 1 \mathrm{DCPD}+\mathrm{C}_{5} \mathrm{H}_{6}$ & 191 & 3.14 & $11.5 \times 10^{3}$ & 3 \\
\hline \multicolumn{5}{|c|}{ Reactions of decomposition to naphthalene } \\
\hline $\mathrm{S} 1 \mathrm{C} 10 \mathrm{H} 9=>\mathrm{S} 2 \mathrm{C} 10 \mathrm{H} 9$ & $1.26 \times 10^{9}$ & 0.90 & $10.7 \times 10^{3}$ & [17] \\
\hline $\mathrm{S} 2 \mathrm{C} 10 \mathrm{H} 9=>\mathrm{S} 1 \mathrm{C} 10 \mathrm{H} 9$ & $7.95 \times 10^{9}$ & 1.03 & $1.8 \times 10^{3}$ & [17] \\
\hline $\mathrm{S} 2 \mathrm{C} 10 \mathrm{H} 9=>\mathrm{S} 3 \mathrm{C} 10 \mathrm{H} 9$ & $7.42 \times 10^{9}$ & 1.02 & $19.3 \times 10^{3}$ & [17] \\
\hline $\mathrm{S} 3 \mathrm{C} 10 \mathrm{H} 9=>\mathrm{S} 2 \mathrm{C} 10 \mathrm{H} 9$ & $1.81 \times 10^{8}$ & 1.34 & $32.7 \times 10^{3}$ & [17] \\
\hline $\mathrm{S} 3 \mathrm{C} 10 \mathrm{H} 9=>\mathrm{S} 4 \mathrm{C} 10 \mathrm{H} 9$ & $8.13 \times 10^{9}$ & 0.90 & $13.1 \times 10^{3}$ & [17] \\
\hline $\mathrm{S} 4 \mathrm{C} 10 \mathrm{H} 9=>\mathrm{S} 3 \mathrm{C} 10 \mathrm{H} 9$ & $2.11 \times 10^{10}$ & 0.80 & $5.9 \times 10^{3}$ & [17] \\
\hline $\mathrm{S} 3 \mathrm{C} 10 \mathrm{H} 9=>\mathrm{S} 11 \mathrm{C} 10 \mathrm{H} 9$ & $2.47 \times 10^{11}$ & 0.72 & $24.9 \times 10^{3}$ & [17] \\
\hline $\mathrm{S} 11 \mathrm{C} 10 \mathrm{H} 9=>\mathrm{S} 3 \mathrm{C} 10 \mathrm{H} 9$ & $2.23 \times 10^{10}$ & 0.46 & $4.9 \times 10^{3}$ & [17] \\
\hline $\mathrm{S} 4 \mathrm{C} 10 \mathrm{H} 9=>\mathrm{S} 10 \mathrm{C} 10 \mathrm{H} 9$ & $7.89 \times 10^{9}$ & 0.90 & $11.3 \times 10^{3}$ & [17] \\
\hline $\mathrm{S} 10 \mathrm{C} 10 \mathrm{H} 9=>\mathrm{S} 4 \mathrm{C} 10 \mathrm{H} 9$ & $4.69 \times 10^{8}$ & 1.34 & $35.1 \times 10^{3}$ & [17] \\
\hline $\mathrm{S} 11 \mathrm{C} 10 \mathrm{H} 9=>\mathrm{S} 10 \mathrm{C} 10 \mathrm{H} 9$ & $8.21 \times 10^{10}$ & 0.28 & $3.3 \times 10^{3}$ & [17] \\
\hline $\mathrm{S} 10 \mathrm{C} 10 \mathrm{H} 9=>\mathrm{S} 11 \mathrm{C} 10 \mathrm{H} 9$ & $6.09 \times 10^{11}$ & 0.71 & $35.6 \times 10^{3}$ & [17] \\
\hline $\mathrm{S} 10 \mathrm{C} 1 \mathrm{OH} 9=>$ naphthalene+R1H & $1.47 \times 10^{10}$ & 0.96 & $14.0 \times 10^{3}$ & [17] \\
\hline naphthalene+R1H=>S10C10H9 & $9.33 \times 10^{8}$ & 1.47 & $10.9 \times 10^{3}$ & [17] \\
\hline
\end{tabular}

\footnotetext{
${ }^{1}$ as for 1,3 -cyclopentadiene $+\mathrm{H}=$ cyclopentadienyl $+\mathrm{H}_{2}[7]$.

${ }^{2}$ as for 1,3-cyclopentadiene $+\mathrm{CH}_{3}=$ cyclopentadienyl $+\mathrm{CH}_{4}[8]$.

${ }^{3}$ as for 1,3 -cyclopentadiene + allyl $=$ cyclopentadienyl + propene [8] .
} 
Table S6: Kinetic parameters used for the formation of indene from the addition of the cyclopentadienyl radical $\left(\mathrm{C}_{5} \mathrm{H}_{5}\right)$ to 1,3-butadiene as shown in Figure $\mathrm{S} 6$ (units: $\mathrm{cm}^{3}, \mathrm{~mol}, \mathrm{~s}, \mathrm{cal}$ ).

\begin{tabular}{|c|c|c|c|c|}
\hline Reaction & A & $\mathrm{n}$ & Ea & ref \\
\hline \multicolumn{5}{|l|}{ Addition } \\
\hline $\mathrm{C}_{5} \mathrm{H}_{5}+\mathrm{C}_{4} \mathrm{H}_{6} \leftrightarrows \mathrm{C}_{5} \mathrm{H}_{5} @ \mathrm{C}_{4} \mathrm{H}_{6}$ & $1.35 \times 10^{00}$ & 3.01 & $6.4 \times 10^{3}$ & 1 \\
\hline \multicolumn{5}{|l|}{ Ring closure } \\
\hline $\mathrm{C}_{5} \mathrm{H}_{5} @ \mathrm{C}_{4} \mathrm{H}_{6} \leftrightarrows \mathrm{C}_{9} \mathrm{H}_{11} @ @ 1$ & $1.00 \times 10^{08}$ & 0.86 & $5.9 \times 10^{3}$ & 2 \\
\hline \multicolumn{5}{|l|}{ Isomerization } \\
\hline $\mathrm{C}_{9} \mathrm{H}_{11} @ @ 1 \leftrightarrows \mathrm{C}_{9} \mathrm{H}_{11} @ @ 2$ & $5.9 \times 10^{12}$ & 0.00 & $28.9 \times 10^{3}$ & 3 \\
\hline $\mathrm{C}_{9} \mathrm{H}_{11} @ @ 2 \leftrightarrows \mathrm{C}_{9} \mathrm{H}_{11} @ @ 1$ & $4.1 \times 10^{12}$ & 0.00 & $46.5 \times 10^{3}$ & 3 \\
\hline \multicolumn{5}{|l|}{ Decomposition } \\
\hline $\mathrm{C}_{9} \mathrm{H}_{11} @ @ 2=>\mathrm{C}_{9} \mathrm{H}_{10} @ @+\mathrm{H}$ & $4.50 \times 10^{12}$ & 0.00 & $20.5 \times 10^{3}$ & 4 \\
\hline $\mathrm{C}_{9} \mathrm{H}_{10} @ @+\mathrm{H}=>\mathrm{C}_{9} \mathrm{H}_{11} @ @ 2$ & $5.50 \times 10^{12}$ & 0.00 & $7.0 \times 10^{3}$ & 4 \\
\hline $\mathrm{C}_{9} \mathrm{H}_{11} @ @ 2$ => indene $+\mathrm{H}_{2}$ & $2.29 \times 10^{12}$ & 0.00 & $43.7 \times 10^{3}$ & 5 \\
\hline
\end{tabular}

${ }^{1}$ as for cyclopentadienyl + 1,3-cyclopentadiene = 8,9,10-trihydrofulvalenyl [18].

${ }^{2}$ as for hex-5-en-1-yl = cyclohexyl [19].

${ }^{3}$ as for $\mathrm{C9H} 9-\mathrm{c5}=>\mathrm{C} 9 \mathrm{H} 9-\mathrm{c} 5 \mathrm{t}$ and $\mathrm{C9H} 9-\mathrm{c} 5 \mathrm{t}=>\mathrm{C9H} 9-\mathrm{c5}$ [12].

${ }^{4}$ as for $\mathrm{C9H9-c5t}=>$ indene $+\mathrm{H}$ and indene $+\mathrm{H}=>$ C9H9-ct [12] .

${ }^{5}$ As for 1,3-cyclohexadiene $=>$ benzene $+\mathrm{H} 2$ [20]. 
Figure S2: Reactions added in the model to account for the formation of toluene from the cyclopentadienyl radical. The scheme is based on the formalism proposed by [10] for the reaction of the methylcyclopentadienyl radical to benzene.

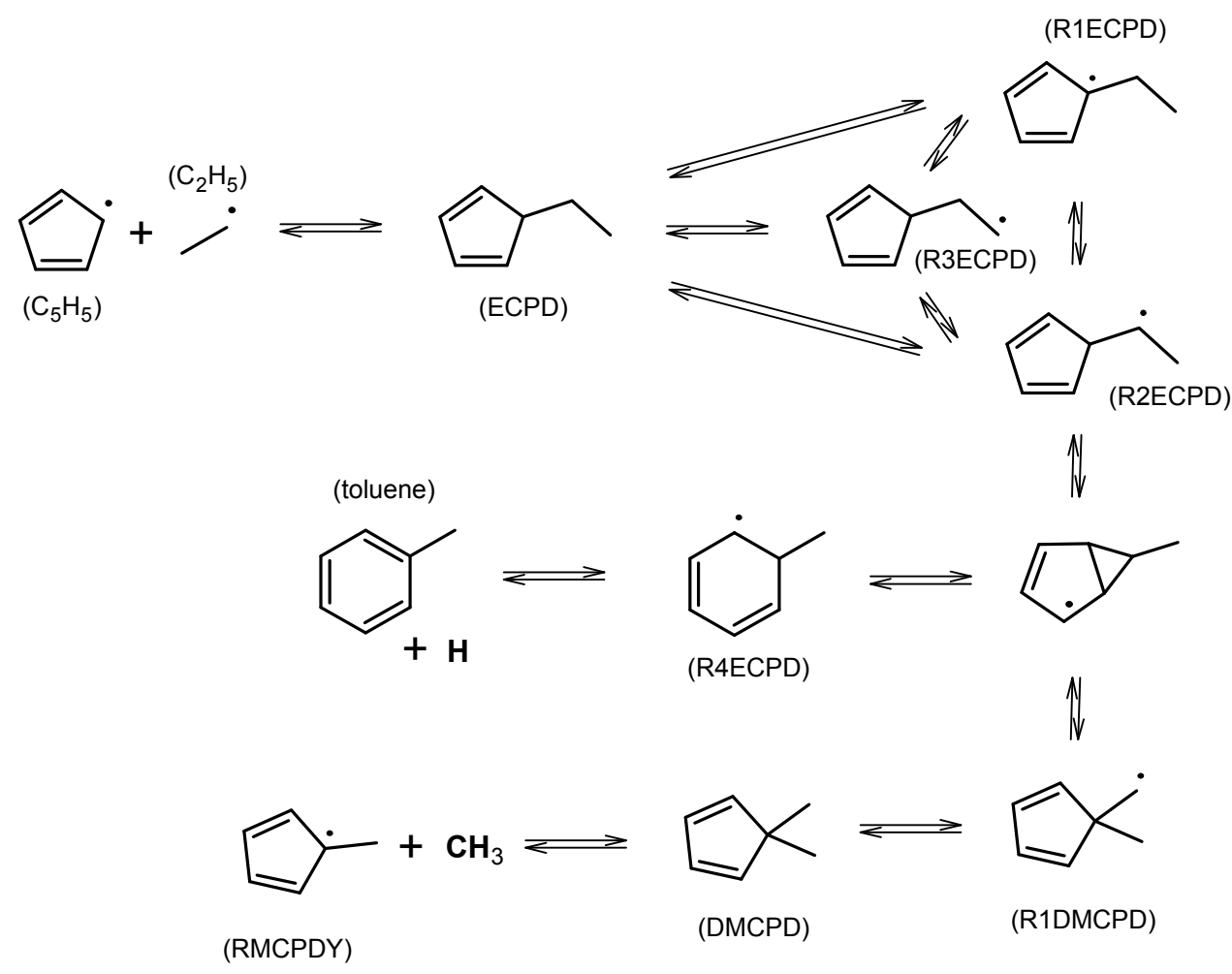


Figure S3: Reactions added in the model to account for the formation of toluene from the reaction of the cyclopentadienyl radical and propene. The first steps are inspired from the study of Fascella et al. about the reaction of the cyclopentadienyl radical and acetylene [12].

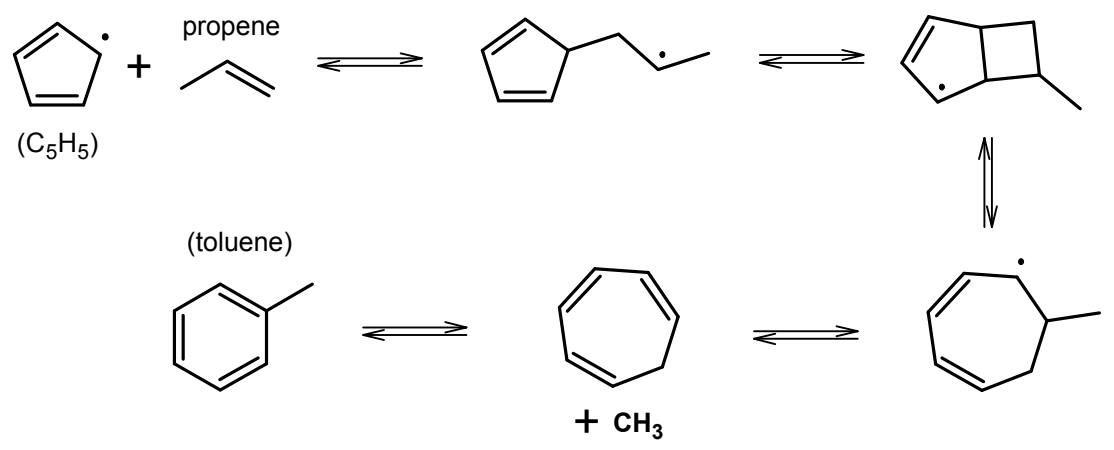


Figure S4: Reactions added in the model to account for the formation of styrene from the cyclopentadienyl radical.

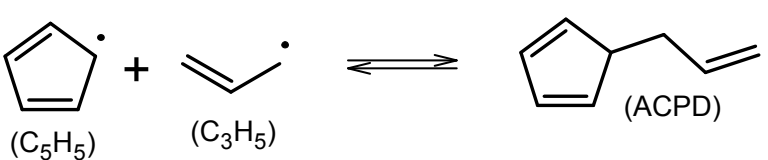

(styrene)<smiles>C#CC=Cc1ccccc1</smiles><smiles>C=CC1C=CC=CC1</smiles>

(R3ACPD)
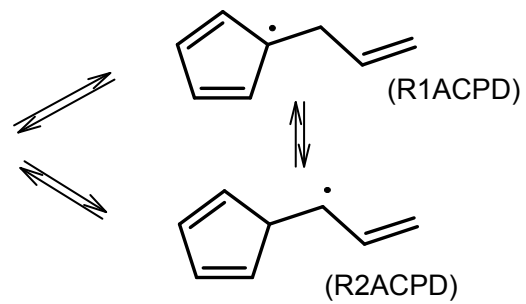

$N$<smiles>C1=CC1</smiles> 
Figure S5: Mechanism proposed by Fascella et al. for the reaction of the cyclopentadienyl radical and acetylene to indene [12].

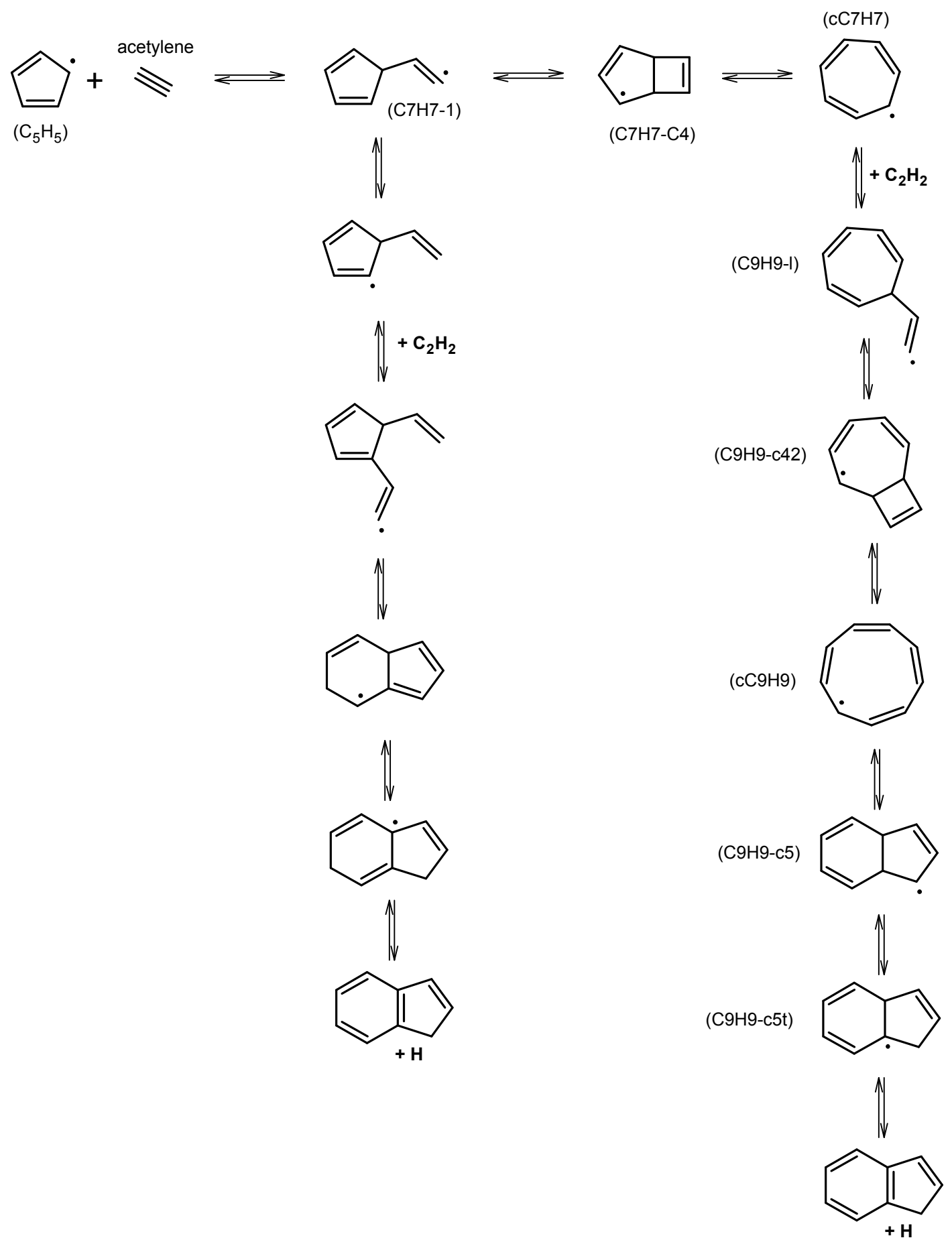


Figure S6: Mechanism used for the formation of benzene from the addition of the cyclopentadienyl radical to 1,3-butadiene

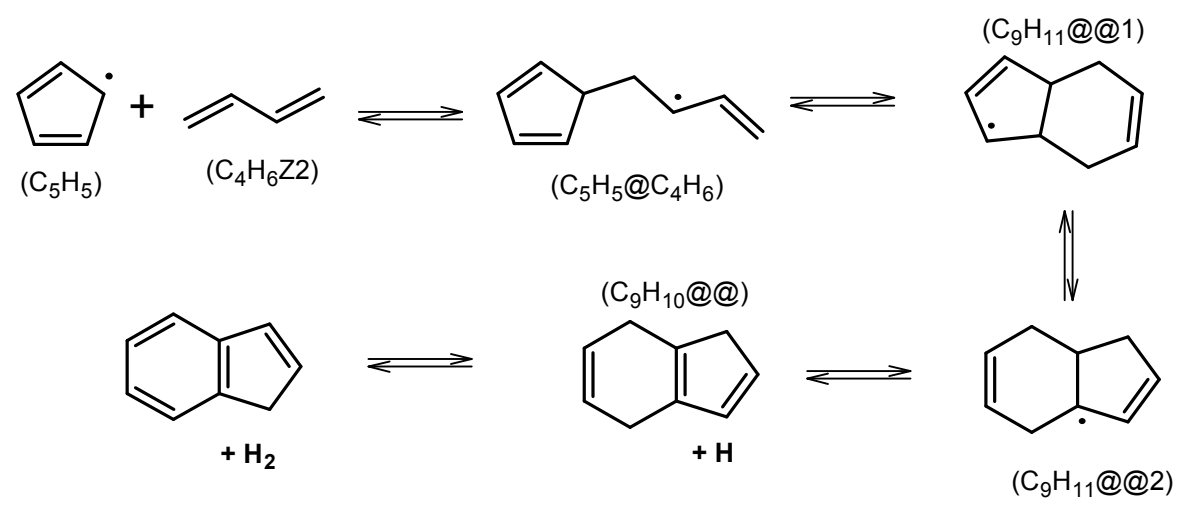


Figure S7: Comparison of the performance of the new model and of that previously published for ethyl-benzene [21].
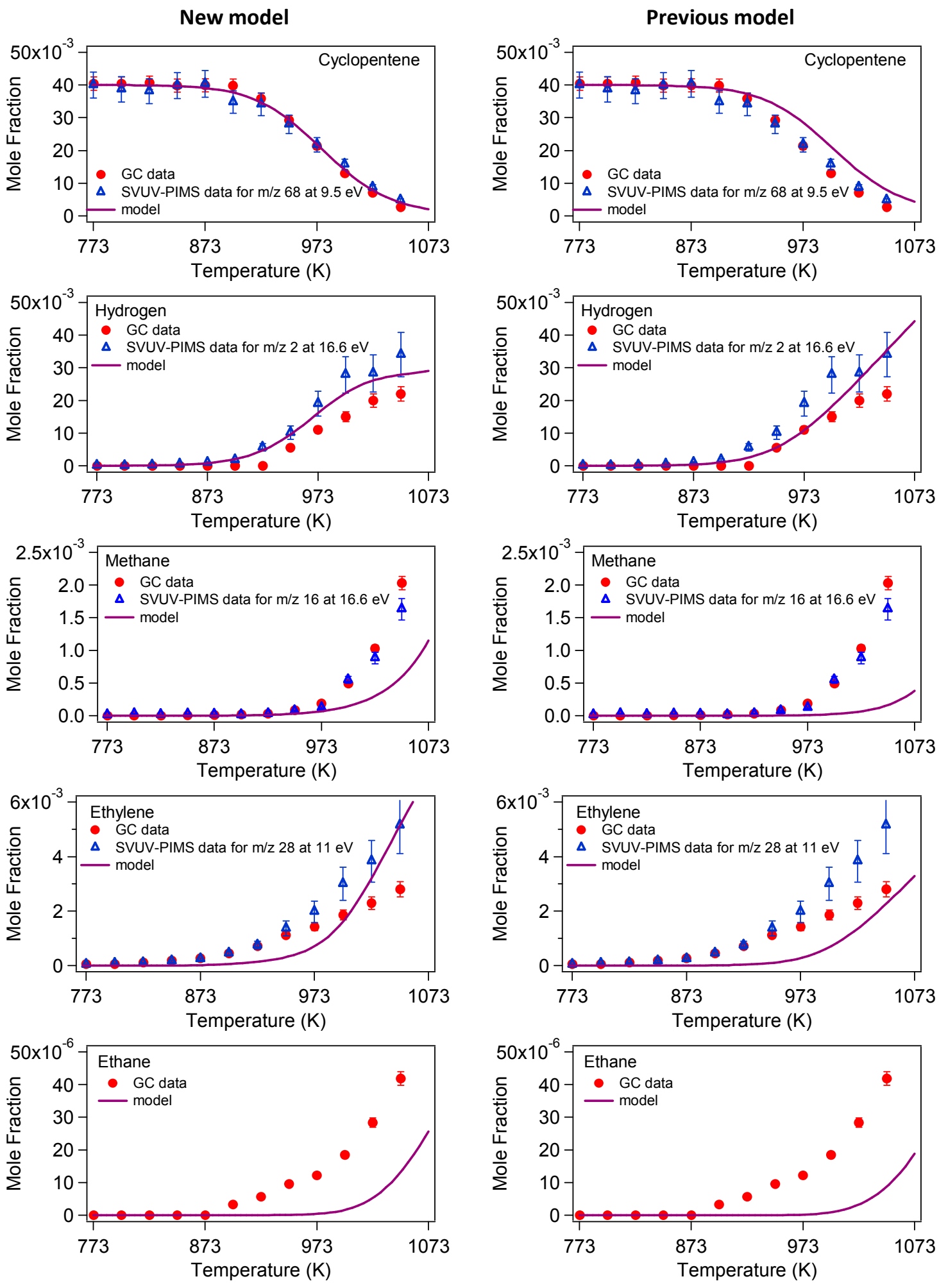
Figure S7 (continued)
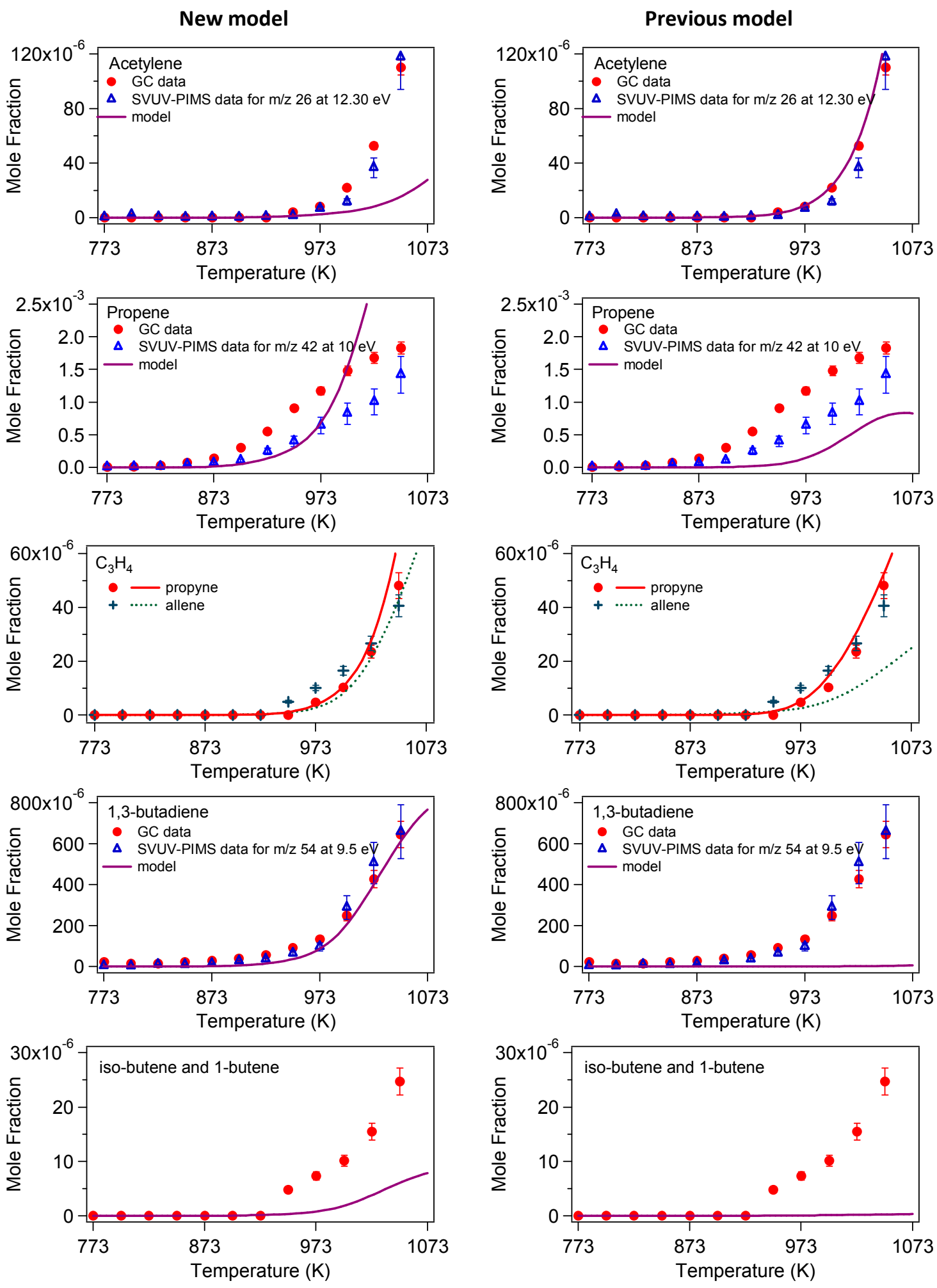
Figure S7 (continued)

\section{New model}
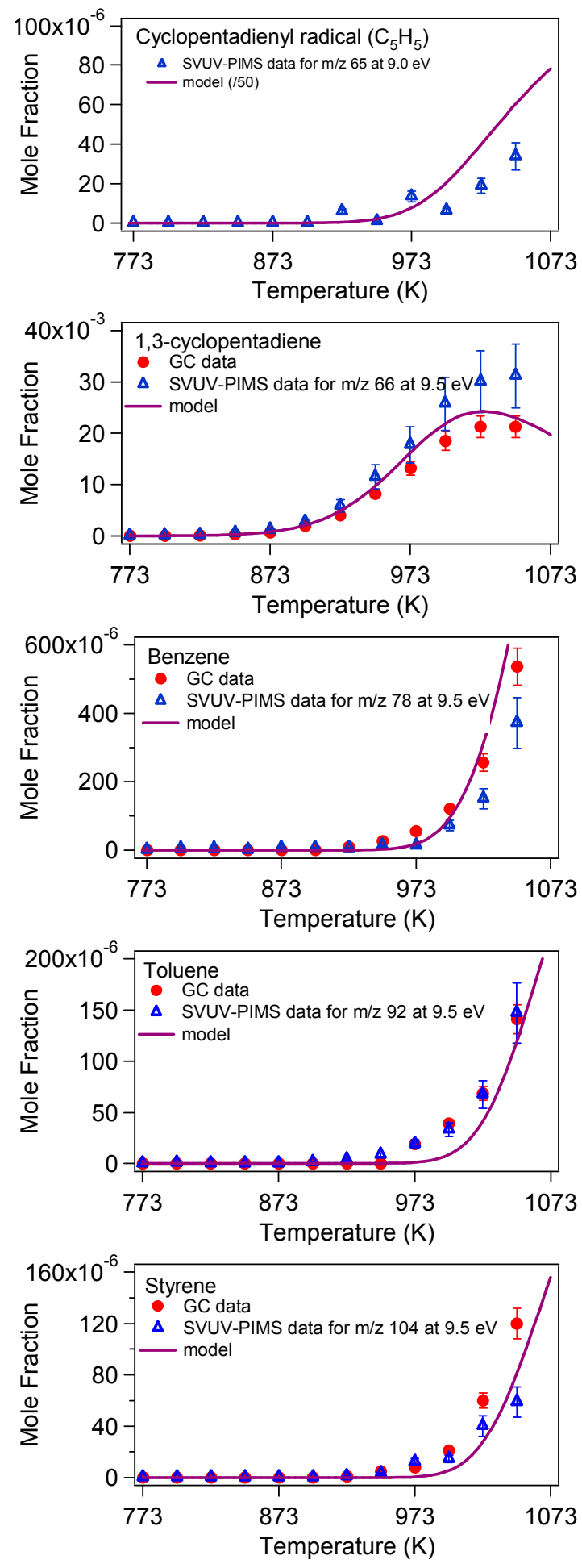

Previous model
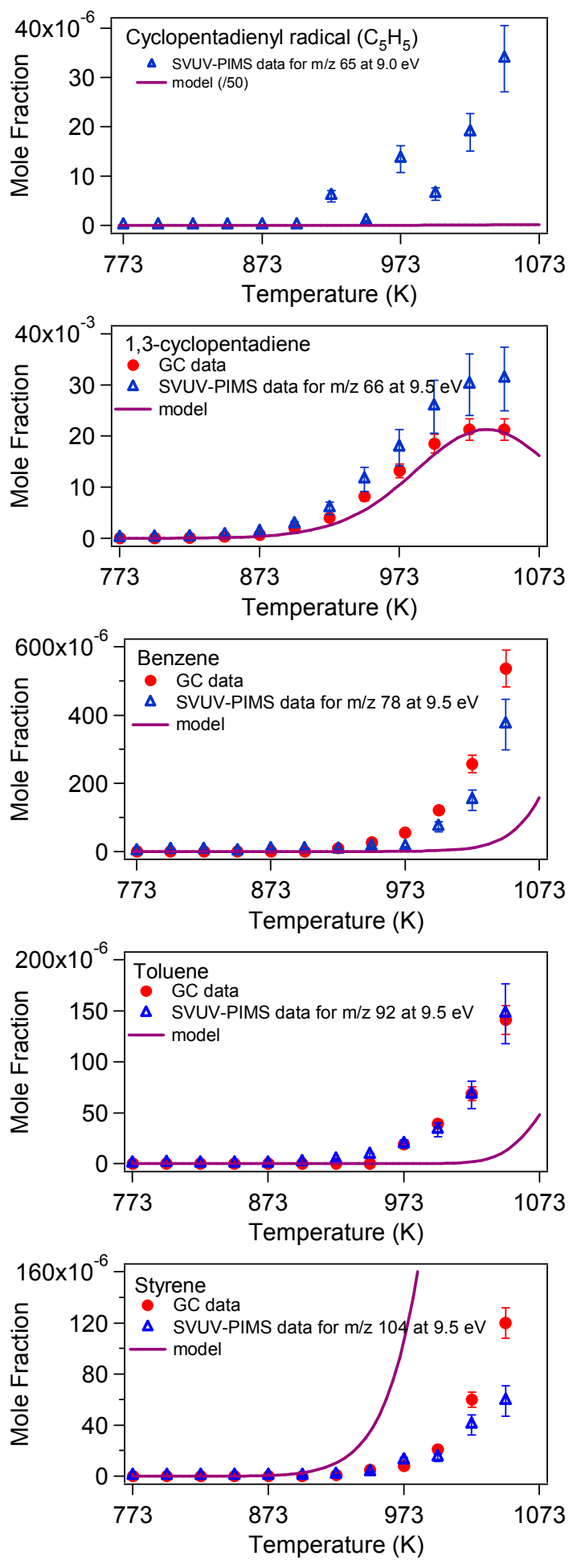
Figure S7 (continued)
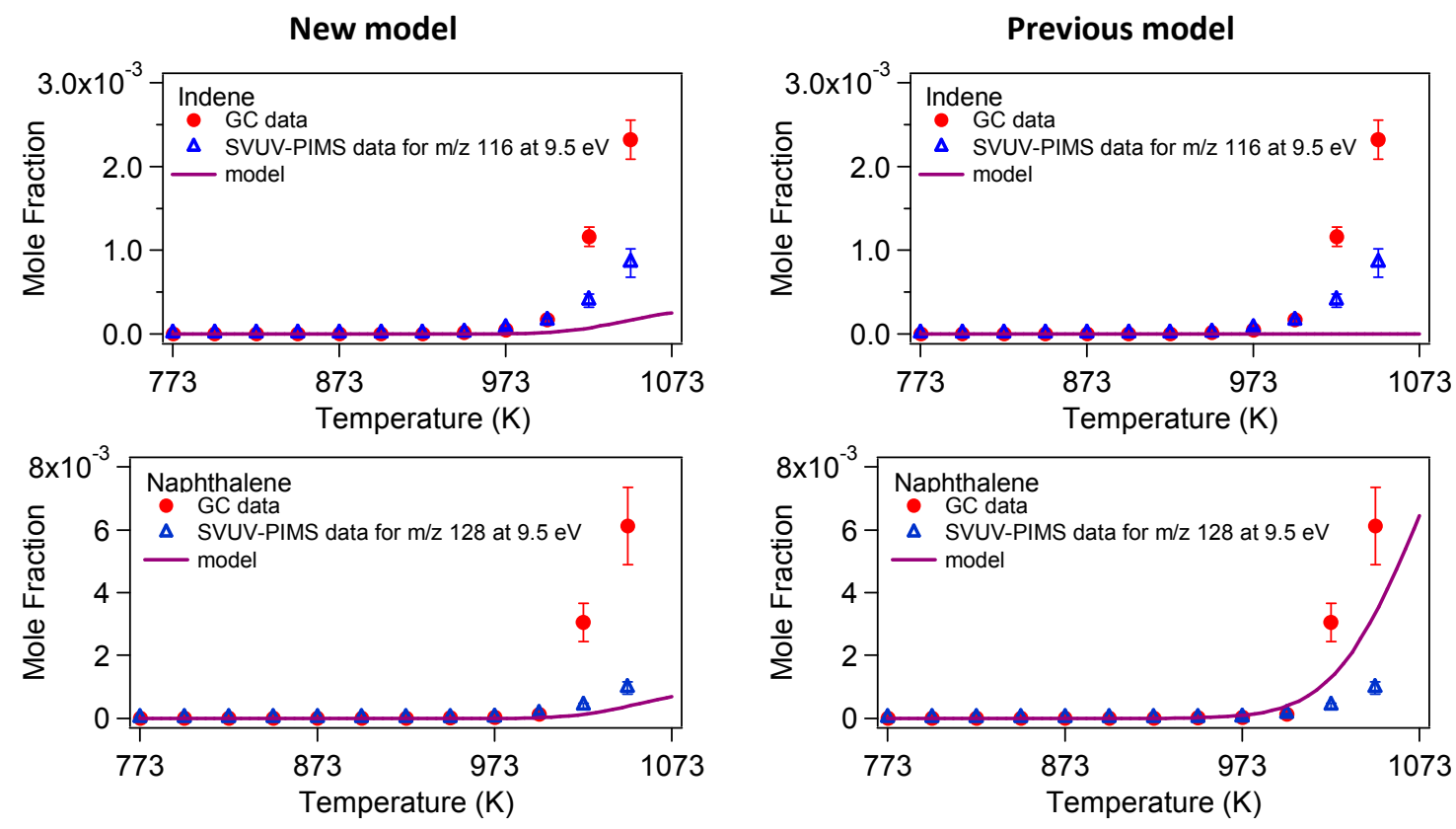


\section{References}

[1] M.J. Frisch, G.W. Trucks, J.R. Cheeseman, G. Scalmani, M. Caricato, H.P. Hratchian, X. Li, V. Barone, J. Bloino, G. Zheng, T. Vreven, J.A. Montgomery, G.A. Petersson, G.E. Scuseria, H.B. Schlegel, H. Nakatsuji, A.F. Izmaylov, R.L. Martin, J.L. Sonnenberg, J.E. Peralta, J.J. Heyd, E. Brothers, F. Ogliaro, M. Bearpark, M.A. Robb, B. Mennucci, K.N. Kudin, V.N. Staroverov, R. Kobayashi, J. Normand, A. Rendell, R. Gomperts, V.G. Zakrzewski, M. Hada, M. Ehara, K. Toyota, R. Fukuda, J. Hasegawa, M. Ishida, T. Nakajima, Y. Honda, O. Kitao, H. Nakai, Gaussian 09, 2009.

[2] J.A. Montgomery Jr., M.J. Frisch, J.W. Ochterski, G.A. Petersson, J. Chem. Phys. 110 (1999) 28222827.

[3] NIST Chem. WebBook (2015).

[4] S. Sharma, W.H. Green, J. Phys. Chem. A 113 (2009) 8871-8882.

[5] A. Burcat, M. Dvinyaninov, Int. J. Chem. Kinet. 29 (1997) 505-514.

[6] J. Biet, M.H. Hakka, V. Warth, P.-A. Glaude, F. Battin-Leclerc, Energy Fuels 22 (2008) 2258-2269.

[7] K. Roy, P. Franck, in:, Proc. 21st Symp. Shock Waves, 1997, pp. 403-407.

[8] K. Wang, S.M. Villano, A.M. Dean, Phys. Chem. Chem. Phys. 17 (2015) 6255-6273.

[9] D. Baulch, C. Cobos, R. Cox, C. Esser, P. Frank, T. Just, J. Kerr, M. Pilling, J. Troe, R. Walker, J. Warnatz, J. Phys. Chem. Ref. Data 21 (1992) 411-734.

[10] F. Dubnikova, A. Lifshitz, J. Phys. Chem. A 106 (2002) 8173-8183.

[11] A.M. Mebel, M.C. Lin, T. Yu, K. Morokuma, J. Phys. Chem. A 101 (1997) 3189-3196.

[12] S. Fascella, C. Cavallotti, R. Rota, S. Carrà, J. Phys. Chem. A 109 (2005) 7546-7557.

[13] S.H. Luu, K. Glänzer, J. Troe, Berichte Bunsenges. Für Phys. Chem. 79 (1975) 855-858.

[14] A.M. Dean, J. Phys. Chem. 89 (1985) 4600-4608.

[15] W. Tsang, J. Phys. Chem. Ref. Data 20 (1991) 221-273.

[16] A.M. Mebel, V.V. Kislov, J. Phys. Chem. A 113 (2009) 9825-9833.

[17] V.V. Kislov, A.M. Mebel, J. Phys. Chem. A 111 (2007) 9532-9543.

[18] V.V. Kislov, A.M. Mebel, J. Phys. Chem. A 112 (2008) 700-716.

[19] D.M. Matheu, W.H. Green, J.M. Grenda, Int. J. Chem. Kinet. 35 (2003) 95-119.

[20] S.W. Benson, R. Shaw, Trans. Faraday Soc. 63 (1967) 985-992.

[21] B. Husson, M. Ferrari, O. Herbinet, S.S. Ahmed, P.-A. Glaude, F. Battin-Leclerc, Proc. Combust. Inst. 34 (2013) 325-333. 